\title{
Dengue virus presence and surveillance in Okinawa (Review)
}

\author{
AKIKAZU SAKUDO ${ }^{1}$, TAKASHI ONODERA ${ }^{2}$, HIDEHARU SHINTANI ${ }^{3}$ and KAZUYOSHI IKUTA ${ }^{4}$ \\ ${ }^{1}$ Laboratory of Biometabolic Chemistry, School of Health Sciences, Faculty of Medicine, University of the Ryukyus, \\ Okinawa 903-0215; ${ }^{2}$ Research Center for Food Safety, Graduate School of Agricultural and Life Sciences, \\ The University of Tokyo, Tokyo; ${ }^{3}$ Faculty of Science and Engineering, Chuo University, \\ Tokyo 112-8551; ${ }^{4}$ Department of Virology, Center for Infectious Disease Control, \\ Research Institute for Microbial Diseases, Osaka University, Osaka 565-0871, Japan
}

Received August 25, 2011; Accepted September 9, 2011

DOI: $10.3892 /$ etm.2011.371

\begin{abstract}
Recent reports have shown that the dengue virus (DENV) is a serious concern worldwide, especially in subtropical areas such as South-East Asia. With the development of transportation systems, the risk of DENV infection spreading is increasing. Since mosquitoes transmit DENV to humans, surveillance of DENV-infected mosquito vectors is the most effective approach for preventing DENV. Okinawa is the only prefecture located in a subtropical region in Japan and historically shows continuous importation of DENV-related mosquito vectors. In this review, we describe the current and historical status of DENV in Okinawa.
\end{abstract}

\section{Contents}

1. Introduction

2. History of dengue disease in Okinawa and Japan

3. Recent condition of dengue disease in Okinawa and the surrounding region

4. Conclusion

\section{Introduction}

A plus-strand RNA virus, the dengue virus (DENV), can be transmitted through mosquito vectors, and these infections lead to dengue fever (DF), dengue hemorrhagic fever (DHF) and dengue shock syndrome (DSS) (1). DHF and DSS are potentially lethal. The risk of developing them is correlated with infection by one of the four DENV serotypes (DENV1 to DENV4) and having antibodies to another DENV serotype

Correspondence to: Dr Akikazu Sakudo, Laboratory of Biometabolic Chemistry, School of Health Sciences, Faculty of Medicine, University of the Ryukyus, 207 Uehara, Nishihara, Okinawa 903-0215, Japan E-mail: sakudo@med.u-ryukyu.ac.jp

Key words: dengue, mosquito, Okinawa, Ryukyu, Taiwan from a previous infection (2). Currently, no specific treatment or vaccine for DENV is available (3).

Recently, reports that DENV has rapidly spread and infected or killed humans have increased (1). In the last 50 years, the incidence has increased by 30 -fold. Infection of DENV is estimated at 50 million cases annually (WHO). Among these, Asia and the Pacific region constitute $75 \%$ of the current global disease burden (3). As DENV spreads beyond national borders, the emergence of DENV is of particular concern to public health. In this review, we report the current and past conditions of dengue disease in Okinawa.

\section{History of dengue disease in Okinawa and Japan}

Okinawa is the southwestern-most prefecture in Japan and consists of a chain of hundreds of islands in the Ryukyu Archipelago, which is over $1,000 \mathrm{~km}$ long (Fig. 1). The location is approximately half-way between Kyushu (the southwest region of Japan's main four islands) and Taiwan. The first reports of dengue disease in Japan were in 1893 and 1903 (4) (Table I). Although the case in 1893 was not diagnosed as dengue disease, the invasion route, epidemic status and disease condition suggest that it was DF. In 1903, Dr Jiro Kawamura diagnosed DF in the Yaeyama Islands. This is thought to be the start of the DF epidemic in Japan (5). Although fever epidemics were frequently found after 1893, that of DF was only found in 1893 and 1904 in the Meiji era (1868-1912). At that time, DF showed weak symptoms and caused a low incidence of death. In addition, severe diseases such as cholera and smallpox hid the DF condition.

In July 1903, DF was first diagnosed in the Yaeyama Islands. Subsequently, DF spread to the Miyako Island and the Okinawa main island in September and October, respectively (6). In 1904, DF was still prevalent in Yaeyama and Miyako Islands and the Okinawa main island, and resulted in 61,901 patients and 16 deaths $(7,8)$.

In the Taisho era (1912-1926), the incidence of DF decreased compared to the Meiji era, whereas in 1916 and 1924 DF epidemics were reported $(9,10)$. In the Showa era (1926-1989), DF incidence was found in Japan in 1931, 1934, 1942, 1943 and 1944, whereas there were no incidences after World War II (1939-1945). In Okinawa, a major outbreak of DF was found on Ishigaki Island in 1930. Then, DF spread to the Miyako Island, 

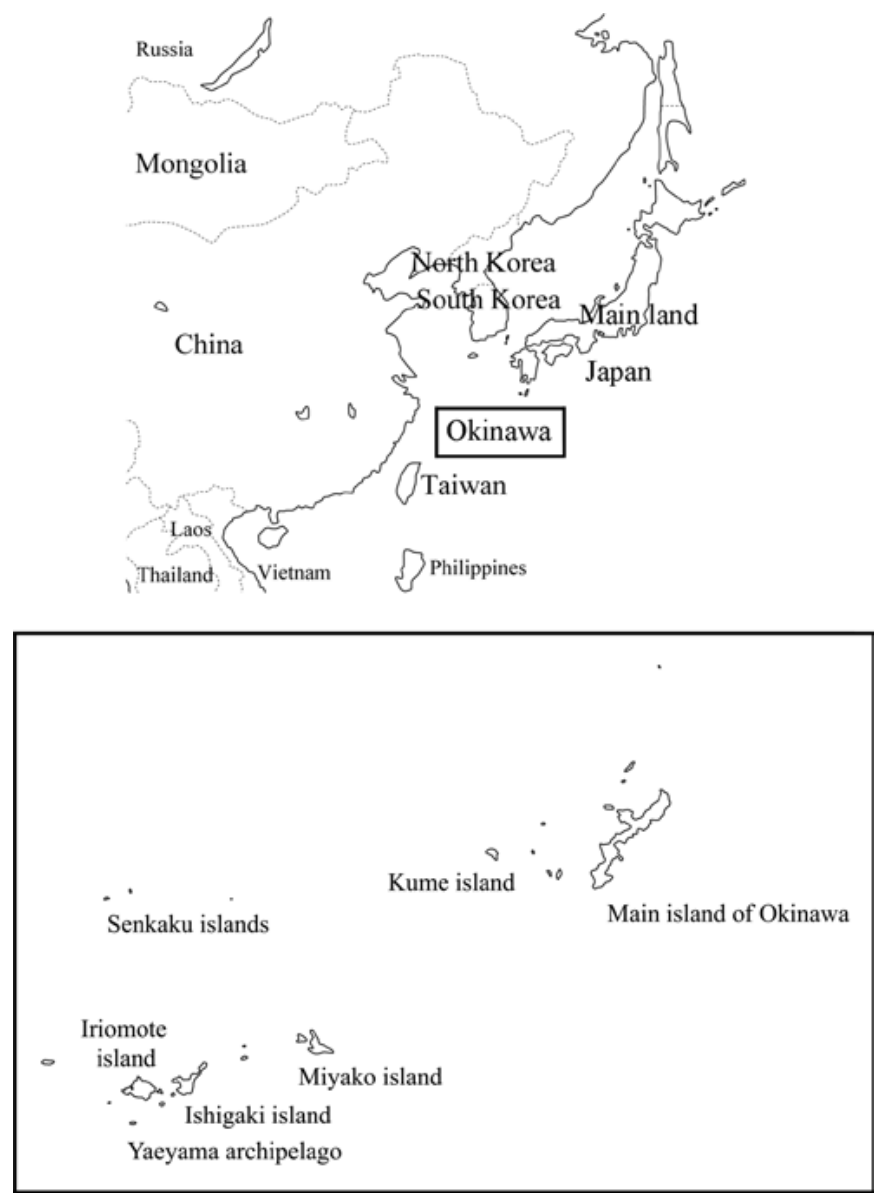

Figure 1. Location of the Okinawa prefecture and Taiwan. Okinawa prefecture is located in the southwest region of Japan. An enlarged illustration of Okinawa Islands is shown in the box.

the Okinawa main island and Kagoshima prefecture on the mainland of Japan (11). After World War II, DF incidence was not found on the main island of Okinawa or the mainland of Japan (12).

In general, the most common invasion route of DF into Okinawa was through the Yaeyama Islands. Then, DF reached the Naha port on the Okinawa main island via the Miyako Island. From Naha, DF spread to other regions of the Okinawa main island and the surrounding islands (13). Therefore, it is reasonable to suggest that most DENV incidents in Okinawa originated from Taiwan, especially in light of mercantile routes of ships during this time. In addition, although Aedes aegypti was previously detected, none was detected after the 1970s in Japan. By contrast, in southern Taiwan, Aedes aegyptia was prevalent. As Taiwan is very close to Okinawa, the continued surveillance for DENV invasion from Taiwan via airplanes and ships is important. Evaluating the distribution of mosquito vectors in this broad region will be useful for the efficient prevention of the appearance and growth of mosquitoes by using chemicals or other methods.

\section{Recent condition of dengue disease in Okinawa and the surrounding region}

Two DENV mosquito vectors (Aedes aegypti and Aedes albopictus) were found during the epidemics in the early
Table I. History of major DF epidemics in Okinawa and Japan (4).

\begin{tabular}{lcc}
\hline Cases & All Japan & Okinawa \\
\hline 1 & 1893 & 1893 \\
2 & 1902 & 1904 \\
3 & 1912 & 1915 \\
4 & 1916 & 1931 \\
5 & 1924 & \\
6 & 1931 & \\
7 & 1934 & \\
\hline
\end{tabular}

20th century in Okinawa and on mainland Japan (14-17). It should be noted that mosquitoes' eggs remain viable for over a year in dry conditions, while the eggs are deposited in the presence of water (3). This allows the mosquito to re-emerge after a cold winter or dry spell. Previous studies have shown that peak mosquito generation was found in the summer season in a pig livestock barn on Yonaguni Island and a public park in Naha City (18). Therefore, close attention should be paid to the mosquito status during the summer season when invasion of DENV is detected in Okinawa or the surrounding region.

An outbreak of DENV3 occurred in southern Taiwan in $1998(3,19)$ and, in 2002, more than 15,000 individuals were infected with DENV in Taiwan. In addition, major dengue outbreaks in Taiwan have been reported (20). Taiwan is located $110 \mathrm{~km}$ from Yonaguni Island, which is the last of the Ryukyu Islands chain and the western-most inhabited island of Japan. In epidemiological studies, a wide variety of DENV strains were isolated from imported and indigenous dengue cases during the outbreak of 1981-2006 in Taiwan (20). The results of molecular epidemiology suggested that constant importation of multiple DENVs via mosquitos and individuals from neighboring southeast Asian countries through close commercial links and air travel was responsible for local outbreaks. In Japan, quarantine stations at airports and harbours have performed surveillance for DENV-infected mosquitoes. However, in Okinawa there are US military bases, which the Japanese government cannot control. Therefore, there is the possibility that DENV-infected mosquitoes may invade Okinawa via military airplanes or ships. In addition, the monitoring of migratory wildfowl is necessary, as many birds come to Okinawa in the spring and autumn. In particular, surveillance on Yonaguni Island is important.

Although there are four distinct serotypes (DENV1 to DENV4), molecular analysis during 2003-2007 showed that the geographic distribution of DENV3 strains and genotypes isolated from southeast Asian countries remained unchanged during 2003-2007, whereas the movement and establishment of new DENV1, DENV2 and DENV4 strains were observed in certain areas of Asia (21). Among them, DENV2 and DENV3 genotypes are frequently associated with severe disease accompanying secondary infection, known as antibody-dependent enhancement (2). Therefore, distribution of DENV-infected mosquitoes, especially DENV2 and DENV3, should be observed closely. Thus, effective and sensitive methods for detecting DENV in mosquitoes are required. 


\section{Conclusion}

Herein, we reviewed the current and past presence of DENV incidence in Okinawa and presented a personal view regarding the future perspectives. During 1838-1955, epidemics of dengue disease were reported 10-20 times in Okinawa. Currently, the incidence of DENV infection has not been reported in Okinawa, and DENV-infected mosquitoes are not resident there. However, several (more than 10) incidences of imported infection cases have been reported annually (22). To prepare for the invasion of DENV, the investigation of resident and generated mosquito vectors in surrounding circumstances should be performed. Although, currently, vector surveillance has focused on airports and harbours, the conditions of public parks, livestock barns and stopovers for migratory birds should be also checked.

\section{Acknowledgements}

This study was supported in part by the Japan Science and Technology Agency and the Ministry of Education, Culture, Sports, Science and Technology (MEXT) of Japan.

\section{References}

1. World Health Organization: http://www.who.int/mediacentre/ factsheets/fs117/en/ (Accessed May 20, 2011).

2. Morens DM: Antibody-dependent enhancement of infection and the pathogenesis of viral disease. Clin Infect Dis 19: 500-512, 1994

3. World Health Organization: Dengue Hemorrhagic Fever Diagnosis, Treatment and Control. 2nd edition. World Health Organization, Geneva, 1997.

4. Inafuku S: The History of Diseases in Okinawa. Daiichi Shobo Co., Ltd., Tokyo, 1995.
5. Ryukyu Shimpo Newspaper. October 1, 5, 7,27, 1903.

6. Ryukyu Shimpo Newspaper. October 1, 17, 27, 1903.

7. Government of the Ryukyu Islands: Okinawan History. Vol. 19 1969.

8. Editorial Committee of History of Taira City: History of Taira City, 1985.

9. Takaka K: Gynecological symptoms of Dengue fever in Okinawa Prefecture in Taisho 4. Reports of Okinawa Medical Association, Okinawa, No. 43, 359-378, 1917.

10. Health and Medical Division, Okinawa Prefecture: Outline of Hygiene in Okinawa, pp63-85, 1924.

11. Yoshino T: Dengue fever in Southern Okinawa. Jpn J Clin Exp Med 20: 943, 1943.

12. The Okinawa Times. April 8, 1960.

13. Ura M: Epidemiology of Dengue Fever in Okinawa. Annual Report of Okinawa Prefectural Institute of Public Health, No. 12, 1978.

14. Hawley W: The biology of Aedes albopictus. J Am Mosq Control Assoc 1: S1-S39, 1988.

15. Gubler DJ and Kuno G: Dengue and Dengue Hemorrhagic Fever. CAB International Publishing, New York, 1997.

16. Gratz NG: Critical review of the vector status of Aedes albopictus. Med Vet Entomol 18: 215-227, 2004.

17. Hotta S: Dengue vector mosquitoes in Japan: the role of Aedes albopictus and Aedes aegypti in the 1942-44 dengue epidemics of Japanese main islands. Med Entomol Zool 49: 276-284, 1998.

18. Taira K, Itokzu K, Kudaka J, Nidaira M, Ohno A and Nakamura M: A survey of mosquitoes as Flavivirus vector in Okinawa prefecture. Annual report of Okinawa Prefectural Institute of Health and Environment 41: 39-44, 2007.

19. Harris E and Roberts G: Typing of dengue viruses in clinical specimens and mosquitoes by single-tube multiplex reverse transcriptase PCR. J Clin Microbiol 36: 2634-2639, 1998.

20. Huang JH and Liao TL: Laboratory-based dengue surveillance in Taiwan, 2005: a molecular epidemiologic study. Am J Trop Med Hyg 77: 903-909, 2007.

21. Shu PY and Su CL: Molecular characterization of dengue viruses imported into Taiwan during 2003-2007: geographic distribution and genotype shift. Am J Trop Med Hyg 80: 1039-1046, 2009.

22. Infectious Disease Surveillance Center: Infectious Agents Surveillance Report. Vol. 25, pp1-2, 2004. 